\title{
Perfil hematológico e bioquímico de pacientes com doença de Chagas atendidos por um serviço de atenção farmacêutica no estado do Ceará
}

\section{Hematological and biochemical profile of patients with Chagas disease attended by a pharmaceutical care service in the state of Ceará}

José Damião da Silva Filho ${ }^{1}$, Alanna Carla Costa ${ }^{2,3}$, Erlane Chaves Freitas ${ }^{3}$, Carlos Eduardo Menezes Viana ${ }^{1}$, Monise Anne Lima$^{3}$, Mônica Coelho Andrade ${ }^{4}$, Laíse dos Santos Pereira ${ }^{3}$, Arduina Sofia Ortet de Barros Vasconcelos Fidalgo ${ }^{2}$, Maria de Fátima Oliveira ${ }^{3,5}$

1. Discente da Pós-graduação em Patologia, pela Universidade Federal do Ceará (UFC), Fortaleza, Ceará, Brasil. 2. Discente da Pós-graduação em Ciências Farmacêuticas da Universidade Federal do Ceará (UFC), Fortaleza, Ceará, Brasil. 3. Laboratório de Pesquisa em doença de Chagas (LPDC), da Universidade Federal do Ceará (UFC), Fortaleza, Ceará, Brasil. 4. Pós-graduação em Ciências Médicas, Universidade Federal do Ceará, Fortaleza, Ceará, Brasil. 5. Docente do Programa de Pós-graduação em Ciências Farmacêuticas pela Universidade Federal do Ceará, Fortaleza (UFC), Ceará, Brasil.

\section{Resumo}

Introdução: A doença de Chagas (DC) é uma parasitose causada pelo agente infeccioso Trypanosoma cruzi. No Brasil, o tratamento específico da DC é realizado com o Benzonidazol (Bz), que devido à sua toxicidade pode levar ao aparecimento de várias reações adversas aos pacientes que fazem uso deste medicamento. Diante disso, existe uma preocupação em avaliar o paciente antes de iniciar a utilização do Bz, a fim de verificar possíveis alterações hematológicas e bioquímicas que eventualmente possam ser atribuídas à utilização deste medicamento. Objetivo: delinear o perfil dos parâmetros hematológicos e bioquímicos de pacientes com DC antes da farmacoterapia com Bz. Metodologia: Estudo retrospectivo que avaliou o perfil sociodemográfico e os parâmetros laboratoriais (eritrograma, leucograma, plaquetas, aspartato aminotransferase - TGO, alanina aminotransferase - TGP, ureia e creatinina) de 89 pacientes chagásicos crônicos atendidos no Serviço de Atenção Farmacêutica ao Paciente com doença de Chagas da Universidade Federal do Ceará no período de janeiro de 2010 a dezembro de 2015. Resultados: A população estudada se caracterizou feminina (52,8\%), média de idade de 53,6 anos, de baixa renda e escolaridade, maioria aposentada, procedente do interior do estado do Ceará. No que se refere às análises hematológicas e bioquímicas, observa-se que todos os parâmetros se encontram dentro dos valores de referência. Na comparação entre gêneros, a análise estatística entre as médias demonstrou diferença significativa nos eritrócitos, no hematócrito, na hemoglobina, nas plaquetas e na contagem de linfócitos. Conclusão: Apesar da evidência de um perfil dentro dos valores normais de referência, o acompanhamento desses parâmetros laboratoriais é de suma importância no seguimento do paciente chagásico, visto que as reações adversas podem aparecer bem no início do tratamento com o Benzonidazol, levando muitas vezes à interrupção do medicamento.

Palavras-chave: Doença de Chagas. Hematologia. Bioquímica

\begin{abstract}
Introduction: Chagas' disease (DC) is a parasite caused by the infectious agent Trypanosoma cruzi. In Brazil, the specific treatment of CD is performed with Benzonidazole (Bz), which due to its toxicity, can lead to the appearance of several adverse reactions to patients who use it. Therefore, there is a certain concern in evaluating the patient before starting the use of Bz, in order to verify possible hematological and biochemical alterations that may be attributed to the use of benzonidazole. Objective: to delineate the profile of the hematological and biochemical parameters of patients with CD before the pharmacotherapy with Bz. Methodology: Retrospective study of sociodemographic and laboratory parameters (erythrogram, leukogram, platelets, aspartate aminotransferase- TGO, alanine aminotransferase - TGP, urea and creatinine) from 89 chronic Chagas' patients treated at the Pharmaceutical Care Service of Federal University of Ceará, the January 2010 to December 2015. Results: The population studied was female (52.8\%), mean age 53.6 years, low income and education, retired majority, from the interior of the state of Ceará. As far as hematological and biochemical analysis are concerned, it is observed that all parameters are within the reference values. In the comparison between genders, statistical analysis between means showed a significant difference in erythrocytes, hematocrit, hemoglobin, platelets and lymphocyte counts. Conclusion: Despite the evidence of a profile within the normal reference values, the follow-up of these laboratory parameters is of paramount importance in the follow-up of the chagasic patient, since adverse reactions may appear well at the beginning of Benzonidazole treatment, often leading to medication interruption.
\end{abstract}

Key words: Chagas disease. Hematology. Biochemistry.

\section{INTRODUÇÃO}

A doença de Chagas (DC) é uma doença tropical negligenciada, endêmica em 21 países, com estimativa de 7-8 milhões de pessoas infectadas, 75-90 Milhões de pessoas em risco de infecção e 14.000 mortes anuais em todo o mundo, principalmente na América Latina ${ }^{1,2,3}$

Nos últimos anos, a DC é uma preocupação global, devido à migração internacional de pessoas de áreas endêmicas para

Correspondência: Alanna Carla Costa. Laboratório de Pesquisa em doença de Chagas (LPDC) da Universidade Federal do Ceará (UFC). Rua Capitão Francisco Pedro, 1210, Rodolfo Teófilo, CEP: 60.430-370, Fortaleza, Ceará, Brasil. E-mail: alannacarla.costa@gmail.com

Conflito de interesse: Não há conflito de interesse por parte de qualquer um dos autores.

Apoio Financeiro: Fundação Cearense de Apoio ao Desenvolvimento Científico e Tecnológico (FUNCAP) e Universidade Federal do Ceará (UFC).

Recebido em: 10 Dez 2016; Revisado em: 3 Mar 2017; 23 Mar 2017; Aceito em: 24 Mar 2017 
não endêmicas, que vem recebendo grande atenção como um problema emergente na América do Norte e Europa. No Brasil, a DC vitimou em média 5.992 pessoas por ano, tendo um aumento significativo das taxas de mortalidade na região Nordeste ${ }^{4}$.

A região Nordeste, ainda representa uma grande preocupação em termos de risco de transmissão da DC. Isso se deve, sobretudo, a três fatores: a região ainda permanece socialmente muito carente e possui os mais altos índices de moradias inadequadas que favorecem à colonização dos triatomíneos nos domicílios; o Nordeste é o epicentro de dispersão das espécies Triatoma brasiliensis e o Triatoma pseudomaculata, espécies de difícil controle pelos meios rotineiros da vigilância entomológica; e nos últimos anos houve redução na cobertura operativa do Programa de Controle da Doença de Chagas em todo o Brasil ${ }^{5,6}$.

No Brasil, atualmente, predominam os casos crônicos decorrentes da infecção por via vetorial em décadas passadas ${ }^{6}$. Diante disso, resta o desafio de prestar assistência aos infectados na fase crônica, dentro da realidade da DC no estado do Ceará. Assim, em 2005, foi criado o Serviço de Atenção Farmacêutica aos pacientes com DC no estado do Ceará, desenvolvido no Laboratório de Pesquisa em Doença de Chagas (LPDC), situado no Departamento de Análises Clínicas e Toxicológicas da Universidade Federal do Ceará (LPDC/DACT/UFC), na cidade de Fortaleza, com o objetivo de oferecer tratamento e acompanhamento aos pacientes crônicos da DC.

No Brasil, o Benzonidazol (Bz) é o único fármaco disponível para o tratamento etiológico da DC. Atualmente, o tratamento é fortemente indicado em crianças com idade igual ou inferior a 12 anos e adolescentes entre 13 e 18 anos com infecção crônica. Nesses casos, o Bz é eficaz na negativação sorológica (seguimento $\geq 5$ anos), na redução da morbimortalidade ou para evitar alterações eletrocardiográficas em curto prazo de evolução. Em adultos, na fase crônica indeterminada, o Benzonidazol é eficaz na redução da morbimortalidade e na progressão da cardiopatia ou na negativação sorológica (seguimento $\geq 5$ anos) ${ }^{7}$.

O Bz é um medicamento tóxico que pode induzir reações adversas, podendo levar à descontinuação do tratamento. Apesar da toxicidade, ainda é o fármaco mais efetivo para o tratamento desse agravo no cenário atual ${ }^{7}$.

Em 2015, os resultados de um estudo prospectivo, multicêntrico e randomizado, denominado BENEFIT, mostrou que a deterioração clínica cardíaca não foi reduzida significantemente com o uso do benzonidazol em pacientes com cardiopatia estabelecida. Portanto, o tratamento etiológico da DC na fase crônica de longa duração, apesar de recomendado é considerado de eficácia incerta especialmente em indivíduos na faixa etária acima de 50 anos. Apesar de o Bz não proporcionar a cura da DC na fase crônica, pode melhorar o perfil de morbimortalidade, melhorando o prognóstico do paciente e, por consequência, aumentando sua sobrevida ${ }^{5,8}$.
Efeitos adversos sérios são relatados em adultos tratados com $\mathrm{Bz}$, incluindo dermatopatia alérgica, manifestações gástricas, neuropatia periférica, ageusia e leucopenia com neutropenia. Essas últimas reações devem-se à depressão da medula óssea que ocorrem entre o 20 e e o 30 dia de tratamento e que em geral a recuperação é sem sequelas. Na monitorização dos eventos adversos cuidados devem ser tomados antes e durante o tratamento. Antes do tratamento, realiza-se exame clínico, hemograma completo, avaliação de enzimas hepáticas e testes de função renal. Esta conduta deve ser repetida no 30 e e 60 dias do início do tratamento. O aparecimento de reações adversas de intensidade moderada ou grave pode muitas vezes levar à interrupção do tratamento. As crianças apresentam menos efeitos adversos do que os adultos e toleram doses mais elevadas ${ }^{7}$.

Portanto, preconiza-se que o paciente chagásico realize exames bioquímicos (função hepática e renal) e hematológicos antes, durante e após o tratamento com Bz, de forma a verificar precocemente as reações adversas associadas ao $\mathrm{Bz}^{7}$. Logo, a importância da realização de exames laboratoriais antes da instituição da farmacoterapia consiste em verificar o estado inicial do paciente assim como também sua capacidade de fazer uso seguro do medicamento, principalmente em pacientes com histórico de patologias anteriores que poderiam possivelmente ser agravadas pelo uso do $\mathrm{Bz}^{7,9}$.

Diante disso, o presente estudo tem como objetivo determinar o perfil hematológico, bioquímico e sociodemográfico de pacientes na fase crônica da DC, antes da farmacoterapia com $\mathrm{Bz}$, atendidos por um serviço de atenção farmacêutica no Estado do Ceará.

\section{METODOLOGIA}

\section{Delineamento e local de estudo}

Trata-se de um estudo observacional descritivo, de caráter retrospectivo, que avaliou parâmetros hematológicos e bioquímicos de pacientes chagásicos por meio da análise de fichas e de exames laboratoriais de pacientes atendidos no Serviço de Atenção Farmacêutica aos Pacientes com Doença de Chagas. $O$ estudo foi desenvolvido no Laboratório de Pesquisa em Doença de Chagas (LPDC) que está situado no Departamento de Análises Clínicas e Toxicológicas da Faculdade de Farmácia, Odontologia e Enfermagem da Universidade Federal do Ceará (DACT/FFOE/UFC). Os exames dos pacientes arquivados no LPDC foram realizados no Laboratório de Análises Clínicas do Hospital Universitário Walter Cantídio (HUWC).

\section{Critérios de inclusão e exclusão}

Foram incluídos pacientes chagásicos crônicos de ambos os sexos que foram atendidos no Serviço de Atenção Farmacêutica aos Pacientes com Doença de Chagas, no período de janeiro de 2010 a dezembro de 2015. Foram excluídos do estudo os pacientes cujos exames não foram arquivados. 


\section{Coleta de dados}

Os dados foram coletados a partir de exames laboratoriais e de fichas de seguimento arquivados no Laboratório de Pesquisa em Doença de Chagas da Universidade Federal do Ceará. Foram coletados dados relativos a sexo, idade, procedência, escolaridade, ocupação, renda familiar. Dos exames laboratoriais, foram coletados dados dos parâmetros hematológicos e bioquímicos antes do tratamento com Benzonidazol.

\section{Análise estatística}

Os dados coletados foram armazenados em forma de tabelas no Microsoft Office Excel 2010. Para o cálculo da análise estatística foi utilizado o programa Graphpad Prism versão 6.0. Para a análise dos dados sociodemográficos realizou-se estatística descritiva. A análise dos parâmetros laboratoriais hematológicos e bioquímicos foi realizada por média e desvio padrão. Na comparação entre gêneros, utilizou-se o teste $t$ para amostras independentes. Uma significância de $p<0,05$ foi adotada para todos os testes.

\section{Aspectos éticos}

O projeto foi aprovado pelo Comitê de Ética em Pesquisa da Universidade Federal do Ceará (COMEPE-UFC), sob o número de parecer 1.063.105 e certificado de Apresentação para apreciação Ética (CAAE) 44163315.1.0000.5054, estando de acordo com as normas do Conselho Nacional de Saúde (Resolução CNS 466/12).

\section{RESULTADOS}

\section{Características sociodemográficas}

Dos pacientes atendidos entre janeiro de 2010 a dezembro de 2015, 89 pacientes foram incluídos no presente estudo por se adequarem aos critérios de inclusão já mencionados.

A Tabela 1 apresenta as características sóciodemográficas e econômicas dos pacientes avaliados no estudo. A população se caracterizou por 42 homens $(47,2 \%)$ e 47 mulheres $(52,8 \%)$. A faixa etária da população variou de 21-84 anos e a média de idade foi de 53,6 anos, com 35,95\% (32) dos pacientes possuindo 60 anos ou mais. Dos pacientes, $65,1 \%$ (58) possuíam o fundamental incompleto ou eram analfabetos. Das ocupações, a mais frequente foi aposentado(a) $(24 ; 27,0 \%)$, seguido por agricultor $(19 ; 21,3 \%)$, e por donas de casa $(13 ; 14,6 \%)$. Relativo à renda familiar, a maioria afirmou ganhar um salário mínimo ou menos $(63 ; 70,8 \%)$.

Em relação à procedência, grande parte da população era proveniente do interior do estado (79; $88,8 \%)$, principalmente da região do Vale do Jaguaribe (50; 56,2\% do total de pacientes), como mostra a Tabela 2.

\section{Parâmetros bioquímicos e hematológicos}

Como observado na Tabela 3, os parâmetros hematológicos e bioquímicos dos pacientes com DC encontram-se dentro dos valores de referência, indicando que esta enfermidade não acarretou alterações neste perfil.

Quando estratificados por sexo (Tabela 4), os parâmetros avaliados permaneceram dentro dos valores de referência. Na comparação dos gêneros, a análise estatística entre as médias demonstrou diferença significativa nos eritrócitos, no hematócrito, na hemoglobina, nas plaquetas e na contagem de linfócitos.

Tabela 1. Características sociodemográficas e econômicas de 89 pacientes chagásicos crônicos atendidos pelo LPDC.

\begin{tabular}{|c|c|c|}
\hline Variáveis & № & $\%$ \\
\hline \multicolumn{3}{|l|}{ Sexo } \\
\hline Masculino & 42 & 47,2 \\
\hline Feminino & 47 & 52,8 \\
\hline \multicolumn{3}{|l|}{ Idade } \\
\hline Menos de 60 anos & 57 & 64,05 \\
\hline Mais de 60 anos & 32 & 35,95 \\
\hline \multicolumn{3}{|l|}{ Escolaridade } \\
\hline Analfabeto & 19 & 21,3 \\
\hline Fundamental incompleto & 39 & 43,8 \\
\hline Fundamental completo & 6 & 6,7 \\
\hline Médio completo & 12 & 13,5 \\
\hline Médio incompleto & 4 & 4,5 \\
\hline Superior completo & 3 & 3,5 \\
\hline Não informado & 6 & 6,7 \\
\hline \multicolumn{3}{|l|}{ Ocupação } \\
\hline Aposentado & 24 & 27,0 \\
\hline Agricultor & 19 & 21,3 \\
\hline Dona de casa & 13 & 14,6 \\
\hline Sem ocupação & 7 & 7,9 \\
\hline Não informado & 7 & 7,9 \\
\hline Outros & 19 & 21,3 \\
\hline \multicolumn{3}{|l|}{ Procedência } \\
\hline Interior do Ceará & 79 & 88,8 \\
\hline Fortaleza & 10 & 11,2 \\
\hline \multicolumn{3}{|l|}{ Renda familiar } \\
\hline < 1 Salário Mínimo & 18 & 20,2 \\
\hline 1 Salário Mínimo & 45 & 50,6 \\
\hline 2 a 4 Salários Mínimos & 26 & 29,2 \\
\hline
\end{tabular}


Tabela 2. Procedência de 89 pacientes chagásicos crônicos atendidos pelo LPDC.

\begin{tabular}{lrr}
\hline Procedência & № & $\%$ \\
\hline Quixeré* & 18 & 20,2 \\
Russas* & 16 & 18,0 \\
Limoeiro do Norte* & 12 & 13,5 \\
Jaguaruana & 11 & 12,4 \\
Fortaleza & 9 & 10,1 \\
Tauá & 7 & 7,9 \\
Alto Santo* & 1 & 4,5 \\
Tabuleiro* & 1 & 1,1 \\
Jaguaribe* & 1 & 1,1 \\
Ipaporanga & 1 & 1,1 \\
Itaiçaba & 1 & 1,1 \\
Palhano* & 1 & 1,1 \\
Outros & 7 & 7,9 \\
Total & 89 & 100,0 \\
\hline
\end{tabular}

*Municípios pertencentes a macrorregião do Vale do Jaguaribe.

Tabela 3. Parâmetros hematológicos e bioquímicos de 89 pacientes chagásicos crônicos atendidos no LPDC.

\begin{tabular}{|c|c|c|}
\hline Exames & Resultados & Valores de Referência \\
\hline \multicolumn{3}{|l|}{ Eritrograma } \\
\hline \multirow[t]{2}{*}{ Hemácias $\left(/ \mathrm{mm}^{3}\right)$} & $4,9 \times 10^{6} \pm 0,4742$ & 4,5 a $6,5 \times 10^{6}$ (Homem) \\
\hline & & 4,0 a $5,5 \times 10^{6}$ (Mulher) \\
\hline \multirow[t]{2}{*}{ Hemoglobina ( $g / d L)$} & $14,28 \pm 1,320$ & 13,5 a 18 (Homem) \\
\hline & & 11,5 a 15,5 (Mulher) \\
\hline \multirow[t]{2}{*}{ Hematócrito (\%) } & $42,87 \pm 3,997$ & 40,0 a 54,0 (Homem) \\
\hline & & 36,0 a 45,0 (Mulher) \\
\hline VCM (fL) & $86,82 \pm 8,057$ & 80 a 96 \\
\hline HCM (pg) & $29,30 \pm 1,759$ & 27 a 32 \\
\hline $\mathrm{CHCM}(\mathrm{g} / \mathrm{dL})$ & $33,34 \pm 1,078$ & 32 a 36 \\
\hline \multicolumn{3}{|l|}{ Leucograma } \\
\hline Leucócitos $\left(/ \mathrm{mm}^{3}\right)$ & $6.689 \pm 1695$ & 4.000 a 10.000 \\
\hline Neutrófilos (/mm³) & $3.777 \pm 1358$ & 1.600 a 7.500 \\
\hline Linfócitos $\left(/ \mathrm{mm}^{3}\right)$ & $2.173 \pm 691,2$ & 800 a 4.500 \\
\hline Monócitos $\left(/ \mathrm{mm}^{3}\right)$ & $460,8 \pm 147,3$ & 80 a 1.000 \\
\hline Eosinofilos $\left(/ \mathrm{mm}^{3}\right)$ & $201,0 \pm 232,0$ & 40 a 600 \\
\hline Plaquetas $\left(/ \mathrm{mm}^{3}\right)$ & $250.142 \pm 60.654$ & 150.000 a 500.000 \\
\hline AST- TGO (U/L) & $22,86 \pm 7,811$ & 13 а 39 \\
\hline ALT- TGP (U/L) & $26,15 \pm 12,93$ & 07 a 52 \\
\hline Ureia (mg/dL) & $31,11 \pm 8,046$ & 15 a 55 \\
\hline \multirow[t]{2}{*}{ Creatinina (mg/dL) } & $1,017 \pm 1,526$ & 0,7 a 1,3 (Homem) \\
\hline & & 0,6 a 1,2 (Mulher) \\
\hline
\end{tabular}

Fonte: Laboratório de Análises Clínicas do HUWC. (VCM- Volume Corpuscular Médio; HCM- Hemoglobina Corpuscular Média; CHCM- Concentração de Hemoglobina Corpuscular Média; AST- Aspartato Aminotransferase; TGOTrasaminase Glutâmico Oxalacética; ALT- Alanina Aminotransferase; TGPTransaminase Glutâmico Pirúvica)
Tabela 4. Parâmetros hematológicos e bioquímicos de homens e de mulheres com doença de Chagas crônica atendimento pelo LPDC.

\begin{tabular}{|c|c|c|c|}
\hline Exame & Feminino & Masculino & $\mathrm{p}$ valor ${ }^{1}$ \\
\hline \multicolumn{4}{|l|}{ Eritrograma } \\
\hline Hemácias $\left(/ \mathrm{mm}^{3}\right)$ & $4,700 \times 10^{6} \pm 0,3894$ & $5,115 \times 10^{6} \pm 0,4673$ & $<0,0001^{*}$ \\
\hline Hemoglobina(g/dL) & $13,64 \pm 0,9725$ & $14,99 \pm 1,300$ & $<0,0001^{*}$ \\
\hline Hematócrito (\%) & $40,98 \pm 2,974$ & $44,94 \pm 3,976$ & $<0,0001^{*}$ \\
\hline VCM (fL) & $87,76 \pm 4,041$ & $85,78 \pm 10,85$ & 0,2519 \\
\hline $\mathrm{HCM}(\mathrm{pg})$ & $29,24 \pm 1,789$ & $29,36 \pm 1,744$ & 0,7461 \\
\hline $\mathrm{CHCM}(\mathrm{g} / \mathrm{dL})$ & $33,31 \pm 1,119$ & $33,37 \pm 1,044$ & 0,8027 \\
\hline \multicolumn{4}{|l|}{ Leucograma } \\
\hline Leucócitos $\left(/ \mathrm{mm}^{3}\right)$ & $6.804 \pm 1747$ & $6.564 \pm 1647$ & 0,5100 \\
\hline Neutrófilos $\left(/ \mathrm{mm}^{3}\right)$ & $3.715 \pm 1363$ & $3.845 \pm 1366$ & 0,6547 \\
\hline Linfócitos $\left(/ \mathrm{mm}^{3}\right)$ & $2.381 \pm 730,8$ & $1.945 \pm 570,7$ & $0,0026^{*}$ \\
\hline Monócitos $\left(/ \mathrm{mm}^{3}\right)$ & $447,2 \pm 156,2$ & $475,7 \pm 137,3$ & 0,3670 \\
\hline Eosinofilos $\left(/ \mathrm{mm}^{3}\right)$ & $154,9 \pm 147,4$ & $251,4 \pm 292,2$ & 0,0506 \\
\hline Plaquetas $\left(/ \mathrm{mm}^{3}\right)$ & $265.222 \pm 66.714$ & $233.626 \pm 48.858$ & $0,0138 *$ \\
\hline AST- TGO (U/L) & $23,47 \pm 9,322$ & $22,21 \pm 5,821$ & 0,4581 \\
\hline ALT- TGP (U/L) & $24,36 \pm 12,57$ & $28,07 \pm 13,17$ & 0,1818 \\
\hline Ureia (mg/dL) & $30,13 \pm 7,026$ & $32,19 \pm 8,997$ & 0,2324 \\
\hline Creatinina (mg/dL) & $1,047 \pm 2,108$ & $0,9833 \pm 0,2439$ & 0,8459 \\
\hline
\end{tabular}

\section{DISCUSSÃO}

Com relação às características sociodemográficas, estudos como de Pinazo et al (2013) e de Ramos et al (2012), observaram um predomínio do gênero feminino, $68,5 \%$ e $63,3 \%$ respectivamente. Já em outros estudos como de Pontes et al (2010) e de Hasslocher-Moreno et al (2012), observaram uma maioria masculina, $65,5 \%$ e $58,9 \%$ respectivamente ${ }^{10,11,12,13}$.

Em relação à média de idade dos participantes do estudo $(53,6$ anos), nota-se semelhança com outros estudos de pacientes com esta mesma patologia, por exemplo, Cândido (2014), que ao avaliar 112 pacientes com DC encontrou uma média de idade de 55,8 anos. Porém, foi relatado um perfil diferente por Viotti et al (2006) que, avaliaram 566 pacientes entre 1984 e 2001 na cidade de Buenos Aires, mostraram uma média de idade de 39,5 anos. Hasslocher-Moreno et al (2012) analisaram 190 pacientes no período de 1986 e 2008, obtiveram uma média de 30 anos. Também foram demonstrados resultados diferentes no estudo de Pontes et al (2010), onde foi relatado uma média de 37 anos para 32 pacientes em Fortaleza-CE $12,13,14,15$.

Os resultados encontrados corroboram com estudos em que os pacientes com DC estão envelhecendo, assim como a população brasileira em geral. Projeções do Instituto Brasileiro de Geografia e Estatística (IBGE) apontam que até 2030 o número de pessoas com mais de 65 anos saltará dos atuais 16 milhões para quase 30 milhões de indivíduos ${ }^{16}$. Em estudo realizado por Viana (2014), demonstrou um aumento significativo nas médias das idades 
dos pacientes chagásicos atendidos no LPDC entre os anos de 2007 e 2013: no ano de 2010,16,92\% dos pacientes atendidos eram idosos; já em 2011, 28,94\%; 2012 foram 37,50\%; por fim, em 2013, 46,15\%. Assim, torna-se compreensível elevada média da idade da população do estudo em questão, visto que isto se dá pelo aumento da entrada de pacientes idosos no serviço ${ }^{17}$.

Quanto à escolaridade, a população desse estudo caracterizouse por um baixo nível educacional. Os resultados corroboram com o estudo realizado por Oliveira et al (2006) onde relataram que $68,6 \%$ dos pacientes possuíam ensino fundamental incompleto. Resultados semelhantes também foram encontrados nos estudos de Correia (2014). Pontes et al (2010) afirmaram que 53\% dos entrevistados possuíam nível fundamental incompleto, corroborando também com o presente estudo que mostrou $46,7 \%$ dos pacientes possuíam ensino fundamental incompleto ${ }^{12,18,19}$.

Quanto à ocupação, resultados de Cândido (2014) corroboram com os do presente estudo, visto que encontrou predominância de aposentados, agricultores e donas de casa com percentuais de $33,0 \%, 20,6 \%$ e $10,7 \%$, respectivamente. Oliveira et al (2006) e Pereira et al (2015) também relataram que as principais ocupações dos pacientes eram aposentados e donas de casa $14,18,20$

Quanto à renda familiar, encontrou-se que $70,8 \%$ dos indivíduos do estudo afirmara ganhar um salário mínimo ou menos, caracterizando a população com baixa renda. Resultados semelhantes também foram encontrados também por Pontes et al (2010) e Correia (2014). Os resultados referentes à renda familiar e à baixa escolaridade comprovam que os pacientes com DC possuem pouco acesso a informações e ao serviço de saúde, o que leva a uma maior importância do acompanhamento desses pacientes por um serviço público especializado na busca da sua melhoria de qualidade de vidaa ${ }^{12,19}$.

A evidência de DC em municípios localizado na região do Vale do Jaguaribe cearense há tempo vem sendo objeto de estudos, como de Freitas et al (2015) e Coutinho et al (2014), respectivamente em Limoeiro do Norte e Russas. Diotaiuti et al.(2000) em Independência - Ceará encontraram uma prevalência global de infecção chagásica em humanos de 5,7\%; Borges-Pereira et al.(2008) encontraram uma soroprevalência da infecção chagásica em 3,1\% no município de Jaguaruana. Vasconcelos (2013) também mostrou em seu estudo a presença de hospedeiros invertebrados infectados nos municípios de Alto Santo, Jaguaribe, Limoeiro do Norte, Quixeré e Tabuleiro do Norte $21,22,23,24,25$.

Em estudo realizado por Cardoso et al (2010) com pacientes que se internaram com Insuficiência Cardíaca congestiva descompensada, provenientes do pronto-socorro do Instituto do Coração do Hospital das Clínicas da Faculdade de Medicina da Universidade de São Paulo e internados no Hospital Auxiliar de Cotoxó observaram que a população chagásica possui algumas médias de parâmetros laboratoriais, como ureia e creatinina, acima dos valores de referência e superiores às do estudo em questão. Possivelmente, isso se deve a insuficiência cardíaca destes pacientes. Dessa forma, uma correlação entre os parâmetros laboratoriais e comorbidades, ou especificamente em pacientes na forma cardíaca, seria interessante em estudos posteriores $^{26}$.

Os parâmetros hematológicos avaliam os elementos celulares do sangue, de forma quantitativa e qualitativa. É o exame complementar mais requisitado nas consultas médicas, a coleta do material é de fácil execução e mostra baixa necessidade de aparato tecnológico, apresentando, portanto, uma boa relação custo benefício sendo indispensável no diagnóstico e no controle evolutivo de várias patologias ${ }^{27}$.

Em algumas doenças crônicas (Tuberculose, bronquiectasia, pneumonia Endocardite, miocardite, osteomielite, Doença de Crohn, Infecção pelo HIV, dentre outras) se evidencia uma anemia associada à diminuição da concentração do ferro sérico e a capacidade total de ligação do ferro, mesmo com concentração do ferro medular normal ou aumentada. Esta anemia é uma síndrome clínica que se caracteriza pelo desenvolvimento deste quadro em doenças inflamatórias crônicas, infecções crônicas e neoplasia. Além disso, há muito tempo, tem-se relatado anemia hipocrômica e microcítica de grau variável em pacientes chagásicos ${ }^{28,29}$. Apesar destes relatos da literatura, no presente estudo não se evidenciou alterações nem na hemoglobina, nem nos índices hematimétricos (VCM, $\mathrm{HCM}, \mathrm{CHCM}$ ).

Quanto às diferenças significativas entre os sexos, nos parâmetros hematológicos, são conhecidas e bem estabelecidas. Os homens possuem valores mais altos para contagem de eritrócitos, hemoglobina e hematócrito que as mulheres. Isso pode ser explicado, em parte, pela influência do hormônio andrógeno na produção eritropoiética e seu efeito na medula óssea. No caso das mulheres, a redução desses valores ocorre pela perda de sangue nas menstruações e pelo pequeno efeito supressor do estrogênio sobre a produção de eritrócitos ${ }^{30,31}$.

Estudos mostram que as mulheres apresentam uma contagem de leucócitos totais, neutrófilos e plaquetas maiores que os homens. Essa diferença é observada em todos os grupos étnicos e aparenta ser de origem biológica, mas a causa ainda é desconhecida. Em estudo anterior, verificou-se que as contagens de plaquetas são significativamente mais elevadas em mulheres com possíveis explicações de compensação para perda de sangue menstrual ou aumento de trombopoietina em mulheres $^{32}$. Já foi demonstrado que a diferença na contagem de leucócitos para as mulheres não pode ser atribuída ao uso de contraceptivos orais ou ao hábito de fumar. Uma explicação baseada na genética é mais provável $\left.\right|^{33}$ e aceita até os dias atuas, visto que há carência de novos estudos que abordem este tema.

Para a verificação do estado geral dos casos de DC, muitas vezes são realizados exames laboratoriais complementares para o seguimento dos casos e do manejo clínico de eventuais 
complicações. No hemograma completo podem ser observados leucopenia ou leucocitose discreta, com desvio à esquerda, associada à linfocitose, bem como eventual anemia hipocrômica e velocidade de hemosedimentação (VHS) moderadamente aumentada. Em casos graves, podem ocorrer plaquetopenia e leucopenia moderadas ${ }^{27}$. No presente estudo essas alterações não foram observadas, uma vez que estes pacientes se encontravam na fase crônica da doença, indicando que nessa fase essas alterações desaparecem, ocorrendo apenas na fase aguda da DC.

Na doença de Chagas também são analisadas as enzimas hepáticas, como exame complementar, são importantes marcadores para verificação do acometimento hepático, especialmente em casos de DC aguda por transmissão oral. As aminotransferases (AST e ALT) frequentemente aparecem elevadas. Bilirrubinas (totais e frações) também podem estar alteradas, com ou sem icterícia visível. O tempo de protrombina (TAP ou TP) prolongado sugere dano hepático. Alterações estas que não foram encontradas no presente estudo ${ }^{34}$.

De forma geral, a explicação mais plausível para esse perfil dentro de valores normais, seria a capacidade do T. cruzi de evadir do sistema imune, em um local inacessível, como no músculo cardíaco ou esquelético. Dessa forma, o parasito provoca uma infecção de caráter crônica, caracterizada por um estado de latência, com replicação mínima do parasita refletindo assim, nos exames laboratoriais dos mesmos ${ }^{35}$.

Segundo o II Consenso Brasileiro em doença de Chagas, os portadores da forma crônica deverão ser atendidos, preferencialmente, nos serviços de atenção básica, recomendando-se, uma vez por ano, a realização de avaliação médica e Eletrocardiograma (ECG) convencional. Caso seja constatada evolução da doença, estes casos deverão ser encaminhados para serviços de saúde de maior complexidade. Enquanto o ECG estiver normal, não se recomenda realizar outros exames complementares de rotina ${ }^{7}$.

\section{CONCLUSÕES}

A população estudada se caracterizou por ser predominantemente feminina, com idade média de 54 anos, de baixa renda e escolaridade, formada em sua maioria por aposentados, agricultores e donas e casa, procedentes em maior parte do interior do estado do Ceará, principalmente da Região do Vale do Jaguaribe.

Em relação ao hemograma, TGO, TGP, ureia e creatinina, se observou contagens e concentrações dentro dos valores tomados como referência, mesmo quando estratificado por sexo, inferindo, possivelmente, a não influência da infecção crônica por T.cruzi nos parâmetros laboratoriais desta população estudada.

Portanto, a avaliação laboratorial de pacientes com DC se faz de grande relevância dada a complexidade de efeitos decorrentes da doença na vida do paciente. Assim, esse contexto inclui tanto a influência do Trypanosoma cruzi, do Bz assim como a presença de comorbidades associadas, que em conjunto representam importantes elementos que podem levar ou não à alterações bioquímicas e hematológicas nos exames dessa população.

\section{AGRADECIMENTOS}

Ao Laboratório de Análises Clínicas do Hospital Universitário Walter Cantídio e à Secretaria de Saúde do Estado do Ceará.

\section{REFERÊNCIAS}

1. World Health Organization (WHO). Chagas disease: Fact sheet. Geneve: Technical Report Series 2013; 304:1-4.

2. Coura JR, Dias JCP. Epidemiology, control and surveillance of Chagas disease - 100 years after its discovery. Mem Inst Oswaldo Cruz 2009; 104 (supl I):31-40.

3. Rassi Jr A, Rassi A, Marin-Neto JA. Chagas disease. Lancet 2010; 375:13881402.

4. Martins-Melo FR, Ramos Junior AN, Alencar $\mathrm{CH}$, Heukelbach J. Multiple causes of death related to Chagas' disease in Brazil, 1999 to 2007. Rev Soc Bras Med Trop. 2012; 45(5): 591-596.

5. Freitas ALC, Freitas SPC, Gonçalves TCM, Lima Neto AS. Vigilância Entomológica dos Vetores da Doença de Chagas no Município de Farias Brito, estado do Ceará. Cadernos de Saúde Coletiva, 2007; 15(2): 231- 240.

6. Dias JCP. Problemas e possibilidades de participação comunitária no controle das grandes endemias no Brasil. Caderno de Saúde Pública. 1998; 14: 19-37.

7. Dias JCP, Ramos Júnior NA, Gontijo ED, Luquetti A, Shiknai-Yasuda MA, Coura JR et al. II Consenso Brasileiro em doença de Chagas, 2015. Epidemiol. Serv. Saúde, Brasília, 2016; 25: 7-86.

8. Morillo CA, Marin-Neto JA, Avezum A, Sosa-Estani S, Rassi A, Rosas F Jr et al. Randomized Trial of Benznidazole for Chronic Chagas' Cardiomyopathy. The New England Journal of Medicine 2015; 373(14): 1295-1306.

9. Comissão Nacional de Incorporação de Tecnologias no SUS (CONITEC). Protocolo Clínico e Doença de Chagas. Ministério da Saúde, Brasília, DF, 2016. $23 \mathrm{p}$.

10. Pinazo MJ, Guerrero L, Posada E, Rodríguez E, Soy D, Gascon J. BenznidazoleRelated Adverse Drug Reactions and Their Relationship to Serum Drug Concentrations in Patients with Chronic Chagas Disease. Antimicrob Agents Chemother. 2013; 570(1): 390-395.

11. Ramos JM, Torrús D, Amador C, Jover F, Pérez-Chacón F, Ponce $Y$ et al. Multicenter epidemiological and clinical study on imported Chagas diseases in Alicante, Spain. Pathogensand Global Health. 2012; 10 (6): 340-345.

12. Pontes VMO, Souza Júnior AS, Cruz FMT, Coelho HLL, Nagao Dias ATN, Coêlho ICB et al. Reações adversas em pacientes com doença de Chagas tratados com benzonidazol, no Estado do Ceará. Rev Soc Bras Med Trop. Uberaba 2010; 43(2).

13. Hasslocher-Moreno AM, Do Brasil PE, De Sousa AS, Xavier SS, Chambela MC, Sperandio Da Silva GM. Safety of benzonidazole use in the treatment of chronic Chagas' disease. J AntimicronChemother. 2012; 67(5): 1261-67.

14. Cândido DS. Avaliação das reações adversas ao tratamento com 
benzonidazol em pacientes chagásicos crônicos atendidos em um servo de atenção farmacêutica no Estado do Ceará [monografia]. Fortaleza: Universidade Federal do Ceará;2014.

15. Viotti R, Vigliano C, Lococo B, Bertocchi G, Petti M, Alvarez MG, Postan M, Armenti A. Long-term Cardiac Outcomes of Treating Chronic Chagas Disease with Benznidazole versus no treatment. Ann Intern Med. 2006; 144: 724-734.

16. IBGE. Instituto Brasileiro de Geografia e Estatística. Projeção da população do Brasil e das Unidades da Federação. Disponível em: <http://www.ibge.gov. br/apps/populacao/projecao/>.Acessado em: 10 de março de 2017.

17. Viana CEM. Perfil socioeconômico e epidemiológico de pacientes chagásicos atendidos no serviço de atenção farmacêutica no Ceará. [monografia]. Fortaleza: Universidade Federal do Ceará;2014.

18. Oliveira FAS, Bicalho GVC, Souza Filho LD, Silva MJ, Gomes Filho Z.C. Características epidemiológicas dos pacientes com Doença de Chagas. Rev Bras Med Fam Comum. 2006; 2(6): 107-13.

19. Correia JPR. Avaliação dos problemas relacionados a medicamentos de pacientes chagásicos em uso de benzonidazol acompanhados por um projeto de atenção farmacêutica em Fortaleza-ce. [monografia]. Fortaleza: Universidade Federal do Ceará; 2014

20. Pereira LS, Freitas EC, Fidalgo ASOBV, Andrade MA, Cândido DS, Silva Filho JD, Michailowsky V, Oliveira MF, Queiroz JAN. Clinical and epidemiological profile of elderly patients with chagas disease followed between 2005-2013 by pharmaceutical care service in Ceará state, Northeastern Brazil. Rev Inst Med trop. S Paulo 2015; 57(2): 145-152.

21.Freitas EC, Oliveira MF, Andrade MA, Vasconcelos ASOB, Silva Filho JD, Cândido DS, Correia JPR, Cruz JNM, Cavalcanti LPG. Prevalence of chagas disease in a rural area in the state of Ceara, Brazil. Rev Inst Med Trop. S. Paulo 2015; 57(5), 431-433.

22. Diotaiuti L, Faria-Filho OF, Carneiro FC, Dias JC, Pires HH, Schofield CJ. Aspectos operacionais do controle de Triatoma brasiliensis. Cad Saude Publica 2000;16 (supl 2):61-67.

23. Coutinho CFS, Santos RS, Teixeira NFD et al. An entomoepidemiological investigation of Chagas disease in the state of Ceará, Northeast Region of Brazil. Cad. Saúde Pública, Rio de Janeiro, 2014; 30(4):785-793.
24. Borges-Pereira J, Sarquis O, Zauza PL, Britto C, Lima MM. Epidemiologia da doença de Chagas em quatro localidades rurais de Jaguaruana, Estado do Ceará. Soroprevalência da infecção, parasitemia e aspectos clínicos. Rev Soc Bras Med Trop 2008;41:345-351.

25. Vasconcelos ASOB. Índice de infestação e infecção de triatomíneos por Trypanosoma cruzi na região sudeste do estado do Ceará. [dissertação de mestrado]. Fortaleza: Universidade Federal do Ceará; 2013.

26. Cardoso J, Novaes $M$, Ochiai M, Regina K, Morgado P, Munhoz R et al. Cardiomiopatia chagásica: prognóstico no perfil clínico-hemodinâmico C. Arq. Bras. Cardiol. 2010; 95(4).

27. Failace, R. Hemograma: manual de interpretação. 4 ed. Porto Alegre: Artmed; 2003.

28. Cançado RD, Chiattone CS. Anemia de Doença Crônica. Rev. Bras. Hematol. Hemoter. 2002; 24(2): 127-136.

29. Howard JE. La enfermedad de Chagas congénita. Colleccion de Monografias Biológicas. № 6. Santiago: Standler Ed; 1962.

30. Menard D, Mandeng MJ, Tothy MB, Kelembho EK, Gresenguet G, Talarmin A. Immunohematological reference ranges for adults from the Central African Republic. ClinDiagn Lab Immunol. 2003; 10:443-445.

31. Morris MW, Davey FR. Exame Básico do Sangue. In: Henry JB. Diagnósticos Clínicos e Tratamento por Métodos Laboratoriais, 19 edição. São Paulo: Manole,1999.

32. Butkiewicz AM, Kemona H, Dymicka-Piekarska V, Matowicka-Karna J, Radziwon P, Lipska A: Platelet count, mean platelet volume and thrombopoietic indices in healthy women and men. Thrombosis Res 2006, 118:199-204.

33. Bain BJ. Ethnic and sex differences in the total and differential white cell count and platelet count. J Clin Pathol. 1996; 49: 664-6.

34. Brasil. Ministério da Saúde. Secretaria de Vigilância em Saúde. Departamento de Vigilância Epidemiológica. Guia de vigilância epidemiológica / Ministério da Saúde, Secretaria de Vigilância em Saúde, Departamento de Vigilância Epidemiológica. - 7. ed. - Brasília : Ministério da Saúde, 2009.

35. Coelho-Castelo AAM, Trombone APF, Rocha CD, Lorenzi JCC. Resposta imune a doenças infecciosas. Medicina, Ribeirão Preto 2009; 42(2): 127-42.

\section{Como citar este artigo/How to cite this article:}

Silva JD Filho, Costa AC, Freitas EC, Viana CEM, Lima MA, Andrade MC, Pereira LS, Fidalgo ASOBV, Oliveira MF. Perfil hematológico e bioquímico de pacientes com doença de Chagas atendidos por um serviço de atenção farmacêutica no estado do Ceará. J Health Biol Sci. 2017 Abr-Jun; 5(2):130-136. 